\title{
Aprepitant, a NK1-antagonist, administered for 16 weeks reduced itch and supported resolution of skin lesions in a patient with chronic prurigo
}

\section{INTRODUCTION}

Chronic prurigo (CPR) results from an "itch-scratch-cycle", with chronic pruritus $(\mathrm{CP})$ and repeated scratching leading to pruriginous nodules.

Substance P and its neurokinin-1 receptor (NK1) play an important role in itch pathophysiology. Aprepitant, a NK1-antagonist, licensed for the treatment of nausea and vomiting during highly emetic chemotherapy, had antipruritic effects in CPR during short-term use. However, longterm itch reduction is required to clear pruriginous skin lesions in CPR.

\section{HISTORY}

We treated a male patient (73y) suffering for more than 20y from recalcitrant CPR, with permanent itch and pruriginous nodules on his extremities and trunk, with aprepitant $80 \mathrm{mg}$ per day for $16 \mathrm{wks}$. Emollients, topical corticosteroids, tacrolimus, and capsaicin, as well as oral antihistamines were without significant effects. Over the years, he repeatedly received UVB, UVA1, oral and bath-PUVA therapies. Finally, after 2 months of saltwater plus narrowband UVB, pruritus was still 4.7 on the VAS ( $0=$ no itch, $10=$ worst imaginable itch $)$ and extensively excoriated pruriginous skin lesions were still present(Fig 1)
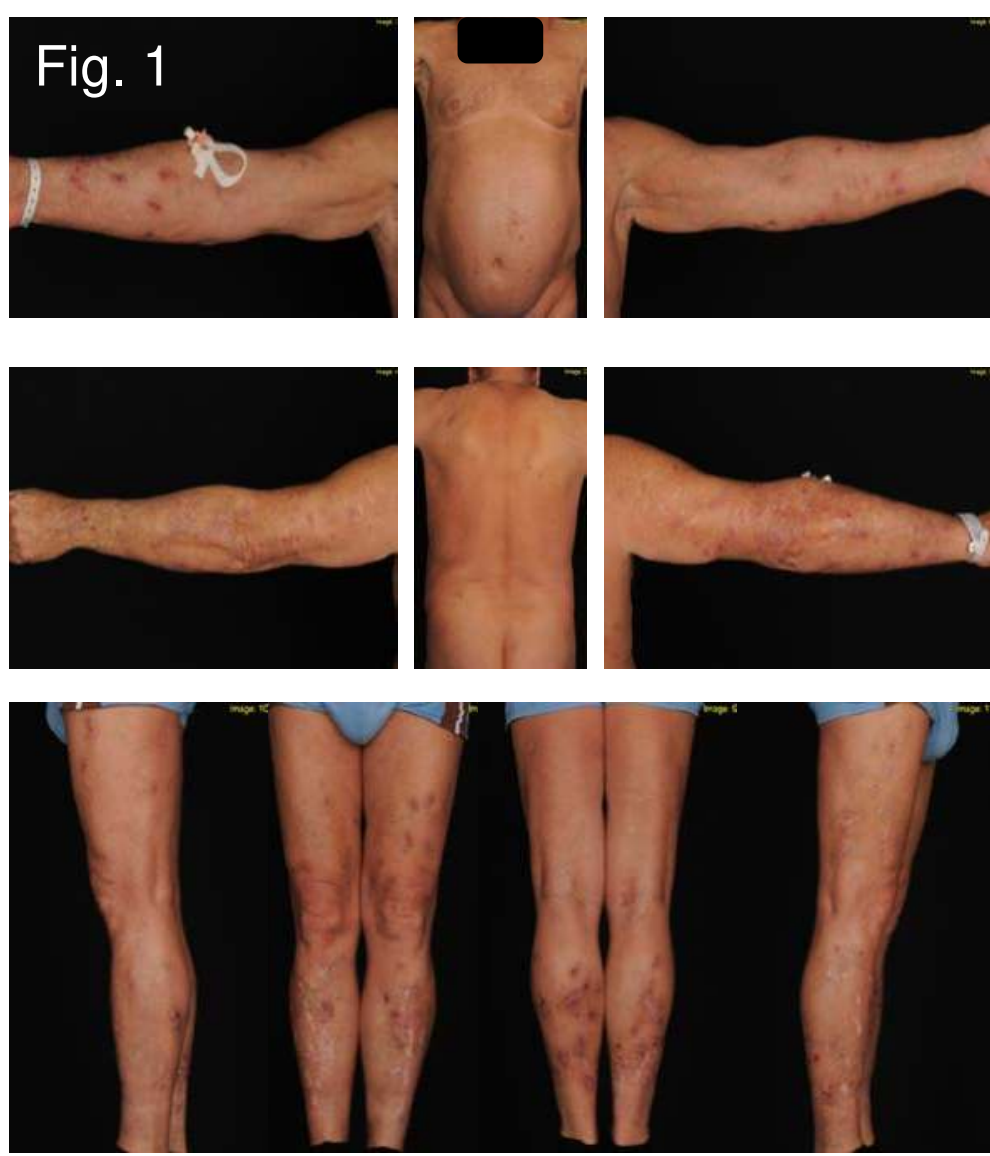

Before Aprepitant,

after saltwater + UVB311 for 2 months
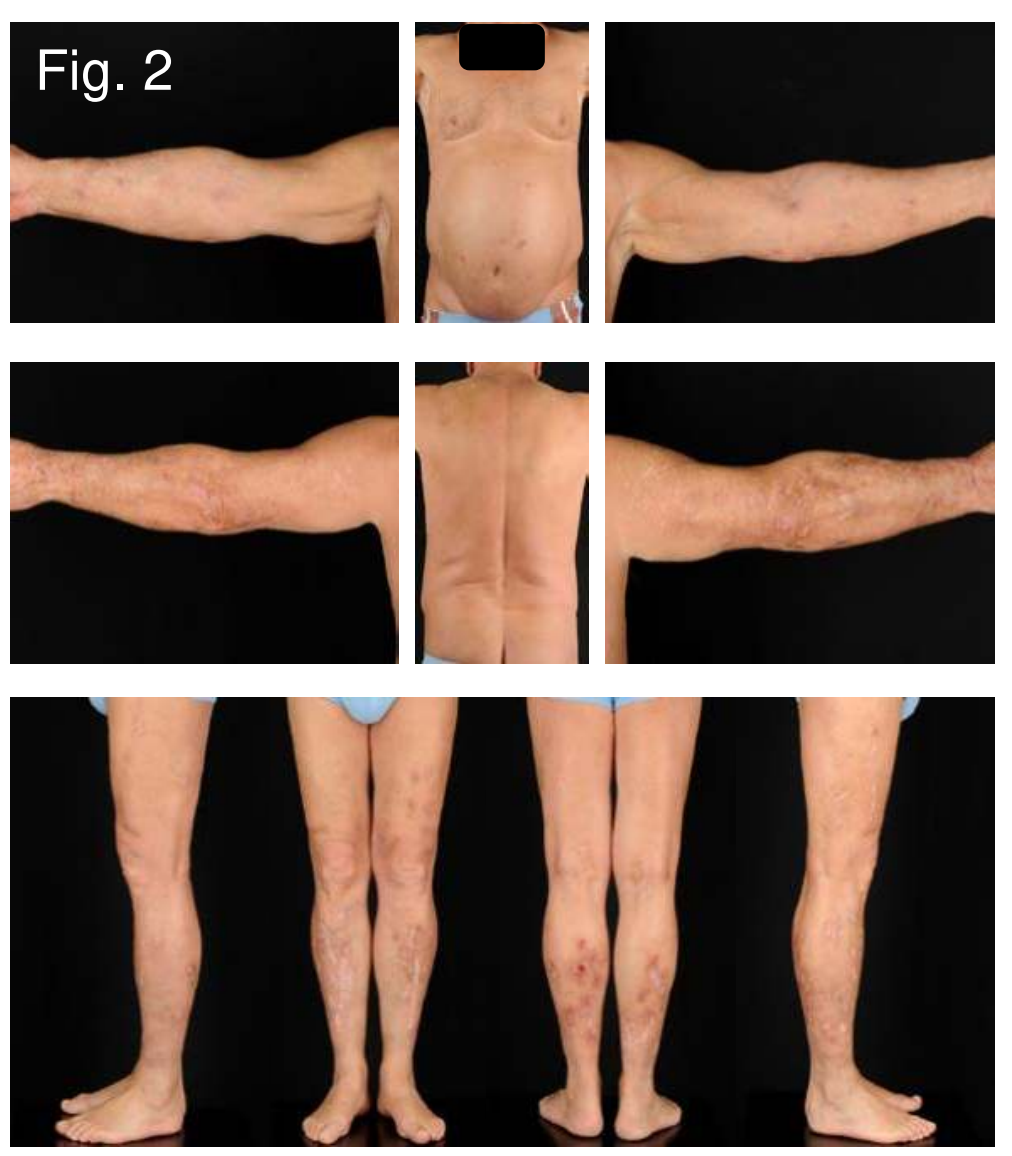

After 16 weeks of Aprepitant
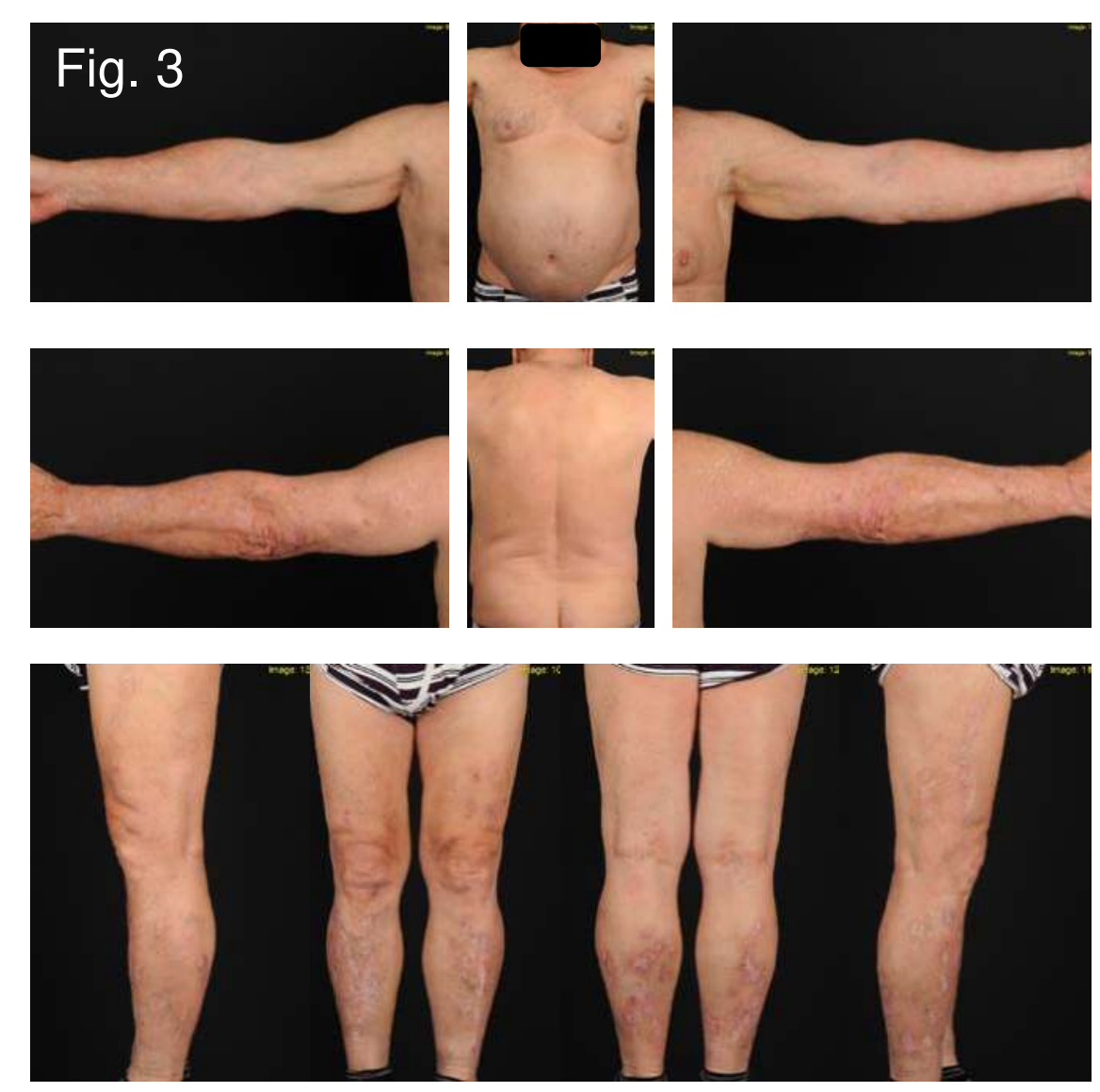

Follow up 1 year after Aprepitant

\section{OUTCOME}

Within 2 wks of daily aprepitant $80 \mathrm{mg}$, pruritus weakened from 4.7 to $3.3(34 \%)$ and was 3.0 after 6 wks. We then added NB-UVB 3x/wk and after further $4 \mathrm{wks}$ topical corticosteroids, once daily for $2 \mathrm{wks}$ and then every other day for 2 wks. This further reduced itch to 2.2. Eventually, due to the high costs of aprepitant, we reduced its dose to $80 \mathrm{mg}$ every other day and stopped it after 2 wks completely (Fig 2). Treatment was continued with NB-UVB, intermittent topical corticosteroids, and daily topical calcipotriol. Within further $8 \mathrm{wks}$ itch was reduced to 0.5 and only very few excoriations remained.

In the following 32 wks pruritus remained low and pruriginous skin lesions tolerable and under control with the use of calipotriol ointment. A short course of topical corticosteroids was necessary during a flare up of lesions after experiencing a transitory ischemic attack 6 months after the end of aprepitant. Thereafter, only skin care was necessary to control the disease (Fig 3).

\section{CONCLUSION}

In conclusion, while various previous treatments were insufficient to permanently reduce itch in our patient, it appears that the long-term (16wks) aprepitant treatment was capable of "breaking the itch-scratchcycle", eventually paving the way for additional UV and topical treatments to become effective in reducing itch and pruriginous lesions

\section{Course of pruritus intensity}

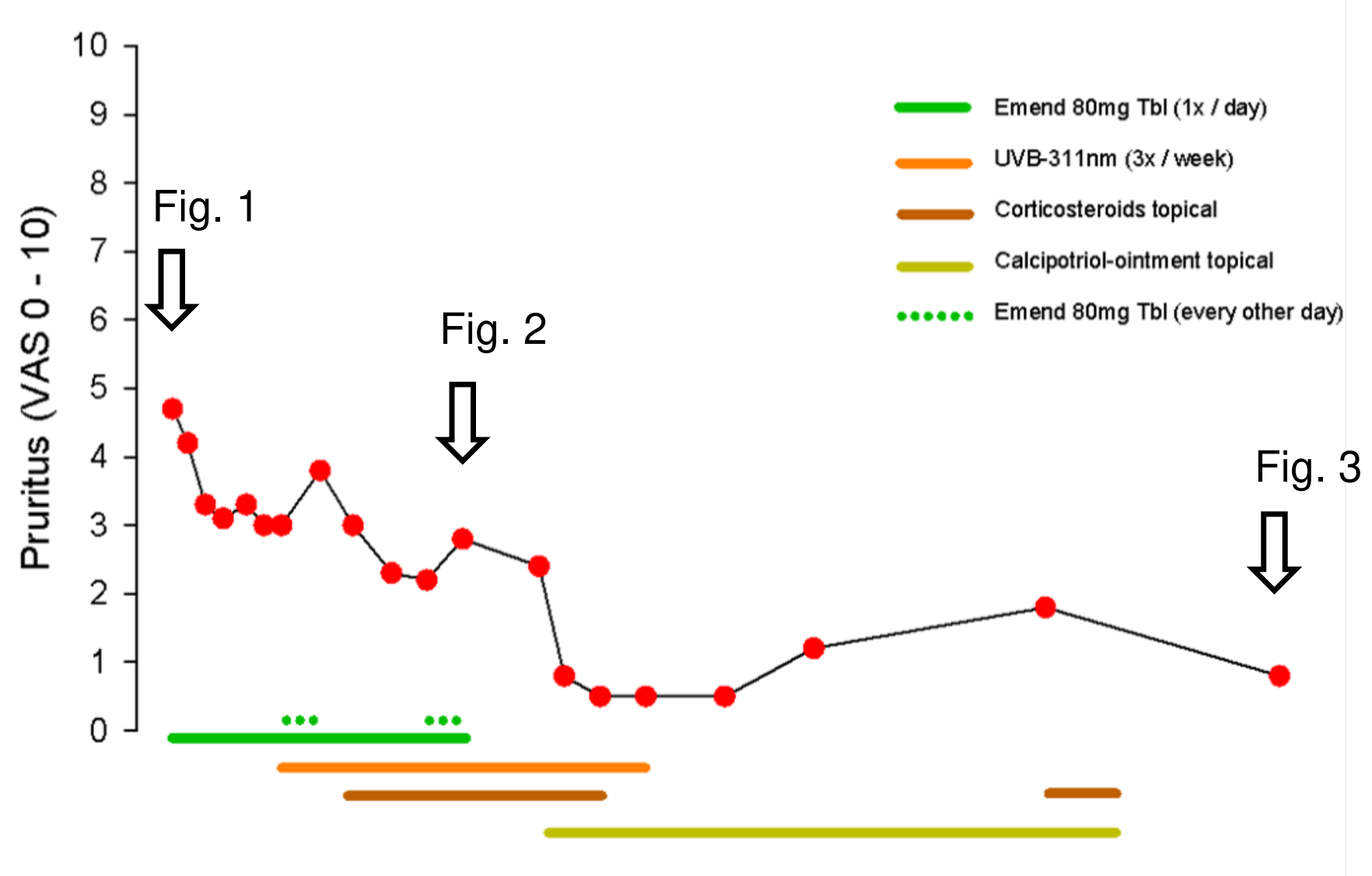

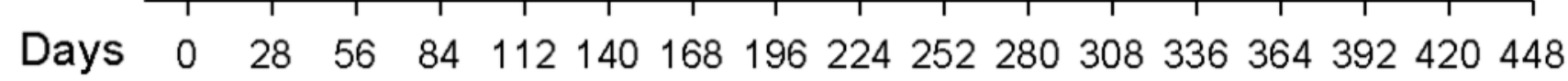

